TIFR/TH/00-42

\title{
LHC Signature of the Minimal SUGRA Model with a Large Soft Scalar Mass
}

\author{
Utpal Chattopadhyay $^{1}$, Amitava Datta ${ }^{2}$, Anindya Datta ${ }^{3}$, \\ Aseshkrishna Datta ${ }^{3}$ and D.P. Roy ${ }^{1}$ \\ ${ }^{1}$ Department of Theoretical Physics, Tata Institute of Fundamental Research, Mumbai - \\ 400 005, India \\ ${ }^{2}$ Physics Department, Visva Bharati, Santiniketan - 731 235, \\ West Bengal, India \\ ${ }^{3}$ Mehta Research Institute, Jhusi, Allahabad - 211 019, India
}

\begin{abstract}
Thanks to the focus point phenomenon, it is quite natural for the minimal SUGRA model to have a large soft scalar mass $\left(m_{0}>1 \mathrm{TeV}\right)$. A distinctive feature of this model is an inverted hierarchy, where the lighter stop has a significantly smaller mass than the other squarks and sleptons. Consequently, the gluino is predicted to decay dominantly via stop exchange into a channel containing $2 b$ and $2 W$ along with the LSP. We exploit this feature to construct a robust signature for this model at the LHC in leptonic channels with 3-4 $b$ tags and a large missing- $E_{T}$.
\end{abstract}


The minimal SUGRA model represents the most attractive model of low energy supersymmetry in terms of simplicity and economy of parameters [1]. Besides it can naturally account for the electroweak symmetry breaking as well as the suppression of flavour changing neutral current effects. The basic parameters of the model are $m_{0}, M_{1 / 2}, A, B$ and $\mu$ - i.e. the soft supersymmetry breaking scalar and gaugino masses, the trilinear and bilinear couplings, along with the supersymmetric Higgs mass parameter. The last two can be determined in terms of the two Higgs vacuum expectation values, $v_{1}$ and $v_{2}$, using the two minimisation conditions. The first one determines the $B$ parameter in term of

$$
v^{2}=v_{1}^{2}+v_{2}^{2}=2 m_{Z}^{2} /\left(g^{2}+g^{\prime 2}\right) \simeq 175 \mathrm{GeV},
$$

and the ratio $v_{2} / v_{1} \equiv \tan \beta$. The second condition gives

$$
\frac{1}{2} m_{Z}^{2}=\frac{m_{H_{1}}^{2}-m_{H_{2}}^{2} \tan ^{2} \beta}{\tan ^{2} \beta-1}-\mu^{2}+\Delta_{R},
$$

where the last term comes from the radiative correction to the Higgs potential.

Thus for any $\tan \beta$, the naturalness of the electroweak scale requires $m_{H_{2}}^{2}$ and $\mu^{2}$ to be of the order of $m_{Z}^{2}$, so that there is no large cancellation between these parameters [2, 3]. Since $m_{H_{2}}^{2}$ is linearly related to the soft mass parameters $m_{0}^{2}$ and $M_{1 / 2}^{2}$ via its RGE, one usually assumes the naturalness criterion to imply $m_{0}$ and $M_{1 / 2}<1 \mathrm{TeV}$ each. Indeed most of the phenomenological works on the minimal SUGRA model are based on this assumption. It has been recently emphasised in ref. [4], however, that for physical values of the top Yukawa and the gauge couplings, $m_{H_{2}}^{2}$ turns out to be practically independent of its GUT scale value $m_{0}^{2}$ for $\tan \beta \gtrsim 5$. Moreover, contrary to some earlier apprehensions, a large value of $m_{0}$ seems to lead to a cosmologically interesting dark matter density [5]. Besides a large $m_{0}$ would also alleviate the potential conflict of the minimal SUGRA model with the electric dipole moments of electron and neutron [6]. Thus the minimal SUGRA model with a large soft scalar mass $\left(m_{0}>1 \mathrm{TeV}\right)$ seems to be attractive both on theoretical and phenomenological grounds. We shall analyse the signature of this model at the large hadron collider (LHC) by exploiting the distinctive characteristics of the large $m_{0}$ limit. Indeed we shall see that they lead to a more robust signal at LHC compared to the canonical SUGRA model.

\section{The Model}

For qualitative understanding of the model it is instructive to look at the approximate expressions for the electroweak scale scalar masses in terms of the universal soft mass parameters at the GUT scale. We shall neglect the GUT scale $A$ parameter, which is unimportant for the present consideration; and assume not too large $\tan \beta$ where the $b$ Yukawa coupling is relatively less significant. Then analytic solutions to the one-loop RGE give

$$
\begin{aligned}
m_{H_{2}}^{2} & =m_{0}^{2}-\frac{3}{2} y m_{0}^{2}+O\left(M_{1 / 2}^{2}\right), \\
m_{U}^{2} & =m_{0}^{2}-y m_{0}^{2}+O\left(M_{1 / 2}^{2}\right) \\
m_{Q}^{2} & =m_{0}^{2}-\frac{1}{2} y m_{0}^{2}+O\left(M_{1 / 2}^{2}\right)
\end{aligned}
$$


where $U$ and $Q$ refer to the 3rd generation singlet and doublet squarks [7]. Here

$$
y=\frac{h_{t}^{2}}{h_{f}^{2}}=\frac{1+1 / \tan ^{2} \beta}{1+1 / \tan ^{2} \beta_{f}},
$$

where the subscript $f$ denotes the fixed point values of the top Yukawa coupling and the corresponding $\tan \beta$ at the electroweak scale. The numerical coefficients of $y$ in (3) simply reflect the corresponding coefficients of $h_{t}^{2}$ in the RG evolutions of $m_{H_{2}}^{2}, m_{U}^{2}$ and $m_{Q}^{2}$ [ 8 .

The top Yukawa coupling is related to its running mass,

$$
h_{t}=m_{t}\left(M_{t}\right) / v \sin \beta \text {, }
$$

which is in turn related to the physical top quark mass $M_{t}$ via

$$
M_{t}=m_{t}\left(M_{t}\right)\left[1+\Delta_{\mathrm{QCD}}+\Delta_{\mathrm{SUSY}}\right] .
$$

The QCD and SUSY radiative corrections add about $6 \%$ and $4 \%$ respectively to the running mass to arrive at the physical top pole mass, $M_{t}=175 \pm 5 \mathrm{GeV}$ [9]. It is well known now that a physical top mass of $175 \mathrm{GeV}$ corresponds to the fixed point value, $\tan \beta_{f} \simeq 1.5$ at the electroweak scale [10], which defines the lower limit of $\tan \beta$ in this model. Of course such a low value of $\tan \beta$ is ruled out by the recent LEP limit on the lightest Higgs boson $\left(h^{0}\right)$ mass [9], suggesting $\tan \beta>2(4)$ for maximal (small) stop mixing. Substituting this value of $\tan \beta_{f}$ in (四) gives

$$
y=\frac{1+1 / \tan ^{2} \beta}{1.44} \simeq \frac{2}{3} \text { for } \tan \beta \gtrsim 5 .
$$

Thus over a large range of $\tan \beta$, which is also favoured by the above mentioned LEP data, one has

$$
m_{H_{2}}^{2} \simeq O\left(M_{1 / 2}^{2}\right)
$$

i.e. the $\mathrm{H}_{2}$ mass at the electroweak scale is practically independent of its GUT scale value $m_{0}$. This is the so called focus point phenomenon [4, 5].

The corresponding values of the 3rd generation squark masses are

$$
\begin{aligned}
& m_{U}^{2} \simeq \frac{1}{3} m_{0}^{2}+O\left(M_{1 / 2}^{2}\right) \\
& m_{Q}^{2} \simeq \frac{2}{3} m_{0}^{2}+O\left(M_{1 / 2}^{2}\right) .
\end{aligned}
$$

The remaining scalar masses are not driven by the top Yukawa coupling, and hence satisfy

$$
m_{R}^{2} \simeq m_{0}^{2}+O\left(M_{1 / 2}^{2}\right)
$$

where the subscript $R$ represents $H_{1}$, sleptons and the squark of the 1st two generations as well as the right-handed bottom squark $\tilde{b}_{R}$. Thus the minimal SUGRA model with large $m_{0}$ implies an inverted mass hierarchy, where $\tilde{t}_{R}$ and to a lesser extent $\tilde{t}_{L}, \tilde{b}_{L}$ are lighter than the 
remaining squarks and sleptons, while all of them are expected to be heavier than the gluino. This in turn implies that gluino should decay dominantly via the lighter stop exchange, i.e.

$$
\tilde{g} \stackrel{\tilde{t}_{1}}{\longrightarrow} t \bar{t} \tilde{\chi}_{i}^{0}, t \bar{b} \tilde{\chi}_{i}^{ \pm} \rightarrow b \bar{b} W W \tilde{\chi}_{1}^{0} X
$$

where $X$ represents any other particles like $Z, h^{0}$ and $W^{+} W^{-}$, which can result from the cascade decay. Thus one expects that the decay of a gluino pair, produced at the LHC, will give a distinctive signal containing $4 b$ quarks and $4 W$ bosons along with a large missing $E_{T}\left(E_{T}\right)$, carried away by the 2 LSPs. Of course some of the signal characteristics like the presence of multiple $b$ quarks are common to many SUSY models having inverted mass hierarchy [11]. However, we feel that the minimal SUGRA with large $m_{0}$ represents by far the most well motivated and well defined model of this kind. A distinctive characteristic of this model is the presence of multiple $W$ bosons in the signal along with $4 b$ quarks. It may be noted here that the LHC signature of this model has been recently considered in ref. [12], which has not discussed however the distinctive characteristics of the model, mentioned above.

\section{The SUSY Spectra}

We have studied the radiative electroweak symmetry breaking and the resulting finetuning parameter in the $m_{0}-M_{1 / 2}$ plane to select optimal values of these parameters and the resulting SUSY Spectra. For this purpose we have modified the radiative electroweak symmetry breaking code of ref. [13], which uses two-loop RGE along with two-loop QCD correction to the top quark mass (eq. 6), by adding the one-loop SUSY correction to the latter [14. Indeed this plays a very significant role in bringing down the focus point to the electroweak scale. The radiative correction to the Higgs potential in eq. (2) is evaluated using the complete one-loop result [15]. The dominant contribution can be written in terms of the average stop mass as

$$
\Delta_{R} \simeq \frac{3 h_{t}^{2}}{16 \pi^{2}} m_{\tilde{t}}^{2}\left[\frac{1}{2}-\ell n\left(\frac{m_{\tilde{t}}}{Q}\right)\right] .
$$

In order to keep this radiative correction small we shall use the minimisation condition (2) at a scale $Q=m_{R}^{\max } / 2$ [13], which is close to the mean stop mass (eqs. 9-11]). The resulting $\mu^{2}$ is then evolved down to the electroweak scale, $Q \simeq m_{Z}$, along with the other mass parameters.

The functional dependence of the electroweak scale on the SUSY parameters is determined via eq. (2). The sensitivity of this scale to fractional variations of these parameters is measured by the sensitivity coefficients

$$
c_{a} \equiv\left|\frac{a}{m_{Z}^{2}} \frac{\partial m_{Z}^{2}}{\partial a}\right|,
$$

where $a$ represents $m_{0}, M_{1 / 2}$ and $\mu$ '. And the fine tuning parameter is defined by the largest of these coefficients

$$
c=\max \left\{c_{m_{0}}, c_{M_{1 / 2}}, c_{\mu}\right\} .
$$

\footnotetext{
${ }^{1}$ As mentioned earlier we set the GUT scale $A$ parameter to zero, since it is unimportant for our purpose. Nonetheless this parameter is generated at the electroweak scale by the RGE, resulting in $\tilde{t}_{L}-\tilde{t}_{R}$ and $\tilde{b}_{L}-\tilde{b}_{R}$ mixing.
} 
It should be noted here that there is an ongoing debate on whether the sensitivity coefficients should include the variation of $m_{Z}^{2}$ with respect to $h_{t}$. On this issue we agree with the authors of ref. [4, 5] that the SM parameters like gauge and Yukawa couplings may have origins totally unrelated to SUSY. Hence, it would not be appropriate to include them in determining the natural ranges of the SUSY parameters.

Fig. 1 shows the contours of this fine-tuning parameter in the $m_{0}-M_{1 / 2}$ plane for $\tan \beta=10$. While the fine-tuning parameter is seen to increase steadily with $M_{1 / 2}$, it is remarkably insensitive to $m_{0}$. In fact for a given $M_{1 / 2} \sim 500 \mathrm{GeV}$, one seems to require by far the least fine-tuning at $m_{0}=1500(2000) \mathrm{GeV}$ for $M_{t}=175$ (180) GeV. The figure also shows the contours of constant $\mu$. The $\mu=100 \mathrm{GeV}$ contour defines the physical boundary, as it represents the lower limit of $\mu$ coming from chargino search at LEP [9. This of course lies close to the no Electroweak Symmetry-breaking boundary, corresponding to $\mu^{2}<0$ [16]. It should be noted that the value of $\mu$ and the resulting physical boundary are very sensitive to the exact value of the top quark mass. We have checked that these values agree quantitatively with those obtained using the recent version of ISASUGRA [17], with the same choice of scale, $Q=m_{R}^{\max } / 2$.

We see from Fig. 1 that the largest values of $M_{1 / 2}$ and $m_{0}$, consistent with a fine-tuning parameter $c<250$ and the physical boundary for $M_{t}=175 \mathrm{GeV}$, are

$$
M_{1 / 2}=500 \mathrm{GeV}, \quad m_{0}=2000 \mathrm{GeV} .
$$

Therefore we choose this point for the computation of the SUSY spectra. Note that the fine tuning parameter at $m_{0}=2000 \mathrm{GeV}$ is about the same as at $m_{0}=200-400 \mathrm{GeV}$. In view of the sensitivity of $\mu$ to the exact value of the top mass, we have shown in Table 1 two sets of SUSY spectra for $M_{t}=175$ as well as $180 \mathrm{GeV}$, leading to $\mu \ll M_{1 / 2}$ and $\mu \sim M_{1 / 2}$ respectively. In order to facilitate comparison with other works these SUSY spectra have been obtained by using the ISASUGRA code [17] with the above mentioned choice of scale. The main difference between the two spectra is that one corresponds to a higgsino LSP, while the other corresponds to a gaugino LSP as in the canonical SUGRA model. We shall analyse the LHC signature for both the cases. It may be added here that although we could have gone to a still larger $m_{0}$ for $M_{t}=180 \mathrm{GeV}$, it would make little difference to the final signature.

\section{The LHC Signature}

It is clear from the SUSY spectra of Table 1 that the dominant SUSY signal at LHC is expected to come from gluino pair-production

$$
g g(q \bar{q}) \rightarrow \tilde{g} \tilde{g} .
$$

We have calculated this leading order cross-section using the CTEQ4M parametrisation [18] at a QCD scale $Q=m_{\tilde{g}}$; and multiplied it by a $K$ factor of 2 to account for the NLO effects [19].

\footnotetext{
${ }^{2}$ To be definitive we have chosen the positive sign of $\mu$ throughout this work; but the results are not expected to be sensitive to this choice.
} 
Since the gluino is expected to dacay dominantly via stop exchange, one has to take account of the Yukawa couplings of top and bottom squarks along with the gauge couplings. The relevant formulae are given ref. [20]. We have used them to calculate the branching ratios of gluino decay. The channels of eq. (12) account for an overall BR of $80 \%$ for both cases. But in the higgsino LSP case a significant fraction of this $(B R \simeq 35 \%)$ corresponds to one of the $W$ 's being off-shell due to the near degeneracy of $\chi_{1}^{ \pm}$and $\chi_{1}^{0}$. The resulting branching fractions of gluino-pair decay into the final states of our interest are shown in Table 2 for both the cases. In the gaugino LSP case (b) the final states containing $4 b 4 W 2 \chi_{1}^{0} \ldots$ have a $B R \simeq 60 \%$, while in the higgsino LSP case (a) the final states containing $4 b 3 W 2 \chi_{1}^{0} \ldots$ have a $B R \simeq 50 \%$ after taking into account the smaller channels not shown in Table 2 . The presence of 3-4 on-shell $W$ bosons lead to distinctive signals in their leptonic decay channels, accompanied by multiple $b$-tags and a large missing- $E_{T}\left(E_{T}\right)$, carried away by the LSP pair. They provide a robust signature for the model at the LHC, with very little SM background, as we see below.

Using a parton level Monte Carlo routine we have computed the signal cross-sections in the isolated $1-l, 2-l$, same sign $2-l$ and 3 - $l$ channels $(l=e, \mu)$, accompanied by $\geq 3$-tags and $E_{T}$. We have also computed the irreducible SM background in these channels from

$$
g g(q \bar{q}) \rightarrow t \bar{t} t \bar{t}
$$

using the MADGRAPH program [21], with $Q=M_{t}$ as the QCD scale.

We have tried to simulate detector resolution by gaussian smearing of the jet and lepton energies [22]

$$
\Delta E_{j} / E_{j}=0.6 / \sqrt{E_{j}}+0.03, \Delta E_{l} / E_{l}=0.15 / \sqrt{E_{l}}+0.01 .
$$

The basic selection cuts are

$$
p_{T}^{j}, p_{T}^{l}, E_{T}>30 \mathrm{GeV},\left|\eta_{j}\right|<3,\left|\eta_{l, b}\right|<2.5,
$$

along with the jet-separation and lepton-isolation cuts

$$
\Delta R_{j j}, \Delta R_{l j}>0.4
$$

where $(\Delta R)^{2}=(\Delta \phi)^{2}+(\Delta \eta)^{2}$. We also require $\geq 3$ b-tags, assuming a tagging efficiency $\epsilon_{b}=0.5$ per $b$-jet [22]. For the $1-l$ channel we require at least two pairs of accompanying jets with $65 \mathrm{GeV}<m_{j j}<95 \mathrm{GeV}$, to simulate two accompanying $W$ bosons. While having very little effect on the signal or the background from (18), it helps to suppress background processes like $t \bar{t} b \bar{b}$, which would otherwise be 7-8 times larger than the signal. For the same reason we require at least one pair of accompanying jets with $65 \mathrm{GeV}<m_{j j}<95 \mathrm{GeV}$ in the $2-l$ channel, but not in the $2-l S S$ (same-sign dilepton) or the 3 - $l$ channels.

Figs. 2 and 3 show the signal and the background cross-sections against the accompanying $E_{T}$ for the 1-l, 2-l, 2-l $S S$ and 3- $l$ channels for $M_{t}=175$ and $180 \mathrm{GeV}$ respectively. Thanks to the accompanying LSP pair, the signal has a much harder $\mathscr{H}_{T}$ distribution than the background in each channel. Thus the signal can be effectively separated from the background by an accompanying $\not_{T}$ cut of $100-200 \mathrm{GeV}$. Such an $\not_{T}$ cut also suppresses other background processes like $t \bar{t} b \bar{b}$ and $W b \bar{b} b \bar{b}$. Therefore the viability of the signature is primarily determined by the signal size. Table 3 lists the signal cross-sections in the four leptonic 
channels with $\geq 3$ b-tags and $\mathscr{H}_{T}>100 \mathrm{GeV}$. Increasing the accompanying $\mathscr{E}_{T}$ cut to 200 $\mathrm{GeV}$ reduces the signal cross-section by only $15 \%$. With the expected annual luminosity of $100 \mathrm{fb}^{-1}$ at the high luminosity run of LHC, one expects to see 30-50 signal events in the single-lepton and 10-20 events in the dilepton channel, with very little SM background. Thus one can unambiguously probe the minimal SUGRA model upto $M_{1 / 2}=500 \mathrm{GeV}$ and the largest allowed value of $m_{0}$.

\section{Summary}

We have considered a minimal SUGRA model with a large $m_{0}=2 \mathrm{TeV}$, which requires no larger fine-tuning than $m_{0}$ of a few hundred GeV. It implies an inverted hierarchy, where the lighter stop has a significantly smaller mass than the other squarks and sleptons. Depending on the exact value of the top quark mass, the LSP can be either gaugino-like or higgsino-like. In either case the inverted hierarchy ensures a robust signature at LHC in the isolated 1-l, $2-l$, same-sign $2-l$ and $3-l$ channels, accompanied by $\geq 3 b$-tags and a large $E_{T}$. Using this signature one can unambiguously probe the model upto $M_{1 / 2}=500 \mathrm{GeV}$ and the largest possible value of $m_{0}$. An investigation of the signature of this model at the Tevatron collider upgrades is currently under progress.

We thank the organisers of WHEPP-6, where this project was started. We also thank Manuel Drees for many helpful communications. Amitava Datta and D.P. Roy acknowledge partial financial support from BRNS project, No. 37/4/97/R \& D II.

\section{References}

[1] H.P. Nilles, Phys. Rep. 110 (1984) 1; H.E. Haber and G.L. Kane, Phys. Rep. 117 (1985) 75.

[2] R. Barbieri and G.F. Giudice, Nucl. Phys. B306 (1988) 63.

[3] G.W. Anderson and D.J. Castano, Phys. Rev. D52 (1995) 1693; Phys. Rev. D53 (1996) 2403; P. Ciafaloni and A. Strumia, Nucl. Phys. B494 (1997) 41; G. Bhattacharya and A. Romanino, Phys. Rev. D55 (1997) 7015.

[4] J.L. Feng, K.T. Matchev and T. Moroi, Phys. Rev. D61 (2000) 075005 [hep-ph/9909334; Phys. Rev. Lett. 84 (2000) 2322 [hep-ph/9908309].

[5] J.L. Feng, K.T. Matchev and F. Wilczek, Phys. Lett. B482 (2000) 388 [hep-ph/0004043].

[6] Y. Kizukuri and N. Oshimo, Phys. Rev. D45 (1992) 1806; J.L. Feng and T. Moroi, hep-ph/9907319; T. Ibrahim and P. Nath, Phys. Rev. D58 (1998) 11301.

[7] M. Carena, M. Olechowski, S. Pokorski and C.E.M. Wagner, Nucl. Phys. B419 (1994) 213.

[8] L.E. Ibanez, C. Lopez and C. Munoz, Nucl. Phys. B256 (1985) 218.

[9] Review of Particle Properties: Euro. Phys. J. C15 (2000) 1. 
[10] J.A. Casas, J.R. Espinosa and H.E. Haber, Nucl. Phys. B526 (1998) 3 [hep-ph/9801365].

[11] H. Baer, M. Drees, C. Kao, M. Nojiri and X. Tata, Phys. Rev. D50 (1994) 2158; H. Baer, P. Mercadante and X. Tata, Phys. Lett. B475 (2000) 289 hep-ph/9912494; V. Barger, C. Kao and R. Zhang, Phys. Lett. B483 (2000) 184 [hep-ph/9911510].

[12] B.C. Allanach, J.P.J. Hetherington, M.A. Parker and B.R. Webber, hep-ph/0005186.

[13] K.L. Chan, U. Chattopadhyay and P. Nath, Phys. Rev. D58 (1998) 096004.

[14] J. Bagger, K. Matchev, D. Pierce and R. Zhang, Nucl. Phys. B49 (1997) 3.

[15] R. Arnowitt and P. Nath, Phys. Rev. D46, (1992) 3981; V. Barger, M.S. Berger and P. Ohmann, Phys. Rev. D49, (1994) 4908.

[16] T. Falk, Phys. Lett. B456 (1999) 171 [hep-ph/9902352].

[17] ISASUGRA (version 7.48): H. Baer, F.E. Paige, S.D. Protopopescu and X. Tata, hep$\mathrm{ph} / 0001086$.

[18] H.L. Lai et al, Phys. Rev. D55 (1997) 1280.

[19] W. Beenakker, R. Hopker, M. Spira and P.M. Zerwas, Nucl. Phys. B492 (1997) 51.

[20] A. Bartl, W. Majerotto, B. Mosslachev, N. Oshimo and S. Stippel, Phys. Rev. D43 (1991) 2214.

[21] T. Stelzer and W.F. Long, Comp. Phys. Comm. 81 (1994) 357.

[22] E. Richter-Was and M. Sapinki, ATLAS note (ATL-PHY-98-132); V. Drollinger, T. Mueller and R. Kinnunen, CMS note (1999/001). 
Table 1: SUSY spectra in $\mathrm{GeV}$ at $m_{0}=2 \mathrm{TeV}, M_{1 / 2}=500 \mathrm{GeV}$, and $\tan \beta=10$, for $M_{t}=$ 175 and $180 \mathrm{GeV}$.

\begin{tabular}{|c|c|c|}
\hline Mass & $\mathrm{M}_{\mathrm{t}}=175 \mathrm{GeV}$ & $\mathrm{M}_{\mathrm{t}}=180 \mathrm{GeV}$ \\
\hline$m_{\tilde{u}_{L}}$ & 2230 & 2227 \\
\hline$m_{\tilde{u}_{R}}$ & 2209 & 2206 \\
\hline$m_{\tilde{d}_{L}}$ & 2231 & 2228 \\
\hline$m_{\tilde{d}_{R}}$ & 2207 & 2204 \\
\hline$m_{\tilde{e}_{L}}$ & 2030 & 2030 \\
\hline$m_{\tilde{e}_{R}}$ & 2010 & 2010 \\
\hline$m_{\tilde{\nu}_{e}}$ & 2029 & 2028 \\
\hline$m_{\tilde{t}_{1}}$ & 1489 & 1424 \\
\hline$m_{\tilde{t}_{2}}$ & 1910 & 1883 \\
\hline$m_{\tilde{b}_{1}}$ & 1902 & 1875 \\
\hline$m_{\tilde{b}_{2}}$ & 2190 & 2188 \\
\hline$m_{\tilde{\tau}_{1}}$ & 1993 & 1993 \\
\hline$m_{\tilde{\tau}_{2}}$ & 2022 & 2022 \\
\hline$m_{\tilde{\nu}_{\tau}}$ & 2021 & 2020 \\
\hline$m_{\tilde{g}}$ & 1283 & 1279 \\
\hline$m_{\tilde{\chi}_{1}^{ \pm}}$ & 119 & 401 \\
\hline$m_{\tilde{\chi}_{2}^{ \pm}}$ & 441 & 567 \\
\hline$m_{\tilde{\chi}_{1}^{0}}$ & 105 & 215 \\
\hline$m_{\tilde{\chi}_{2}^{0}}$ & 134 & 402 \\
\hline$m_{\tilde{\chi}_{3}^{0}}$ & 225 & 541 \\
\hline$m_{\tilde{\chi}_{4}^{0}}$ & 441 & 568 \\
\hline$m_{h}$ & 118 & 121 \\
\hline$m_{H}$ & 2009 & 2077 \\
\hline$m_{A}$ & 2007 & 2075 \\
\hline$m_{H^{ \pm}}$ & 2010 & 2078 \\
\hline$\mu$ & 129 & 550 \\
\hline
\end{tabular}


Table 2: Effective branching ratios for gluino pairs decaying into different channels for $M_{t}$ $=$ (a) $175 \mathrm{GeV}$ and (b) $180 \mathrm{GeV}$, with $\tan \beta=10$. For (a) the generic final state is denoted by $4 b+2 W+2 \widetilde{\chi}_{1}^{0}+X$, and for (b) it is $4 b+4 W+2 \widetilde{\chi}_{1}^{0}+X^{\prime}$. Only those channels having branching ratios greater than $1 \%$ are shown.

\begin{tabular}{|c|c|c|c|}
\hline \multicolumn{2}{|c|}{$M_{t}=175 \mathrm{GeV}$} & \multicolumn{2}{c|}{$M_{t}=180 \mathrm{GeV}$} \\
\hline \hline $\mathrm{X}$ & Br. & $\mathrm{X}^{\prime}$ & Br. \\
\hline \hline$W W$ & 0.0420 & 0 & 0.0412 \\
\hline$W^{*} W^{*}$ & 0.1271 & $Z$ & 0.0595 \\
\hline$W^{*} W$ & 0.1464 & $h$ & 0.0914 \\
\hline$Z^{*} W W$ & 0.0521 & $Z Z$ & 0.0223 \\
\hline$Z^{*} W^{*} W$ & 0.0906 & $h h$ & 0.0594 \\
\hline$Z^{*} Z^{*} W W$ & 0.0163 & $h Z$ & 0.0752 \\
\hline$W^{*} W W W$ & 0.0238 & $W W$ & 0.0743 \\
\hline$W^{*} Z^{*} W W W$ & 0.0147 & $h h Z$ & 0.0165 \\
\hline$W^{*} W^{*} W W$ & 0.0414 & $W W h$ & 0.0823 \\
\hline- & - & $W W Z$ & 0.0536 \\
\hline- & - & $W W W W$ & 0.0334 \\
\hline
\end{tabular}

Table 3: Signal cross-sections for the $1-l, 2-l$, same sign $2-l$, and $3-l$ channels, accompanied by $\geq 3 \mathrm{~b}$-tags and $E_{T}>100 \mathrm{GeV}(\tan \beta=10)$.

\begin{tabular}{|c|c|c|}
\hline & $\begin{array}{c}M_{t}=175 \mathrm{GeV} \\
\text { in fb }\end{array}$ & $\begin{array}{c}M_{t}=180 \mathrm{GeV} \\
\text { in fb }\end{array}$ \\
\hline$\sigma_{1}$ & 0.303 & 0.55 \\
\hline$\sigma_{2}$ & 0.094 & 0.22 \\
\hline$\sigma_{2}(\mathrm{SS})$ & 0.047 & 0.1 \\
\hline$\sigma_{3}$ & 0.013 & 0.06 \\
\hline
\end{tabular}



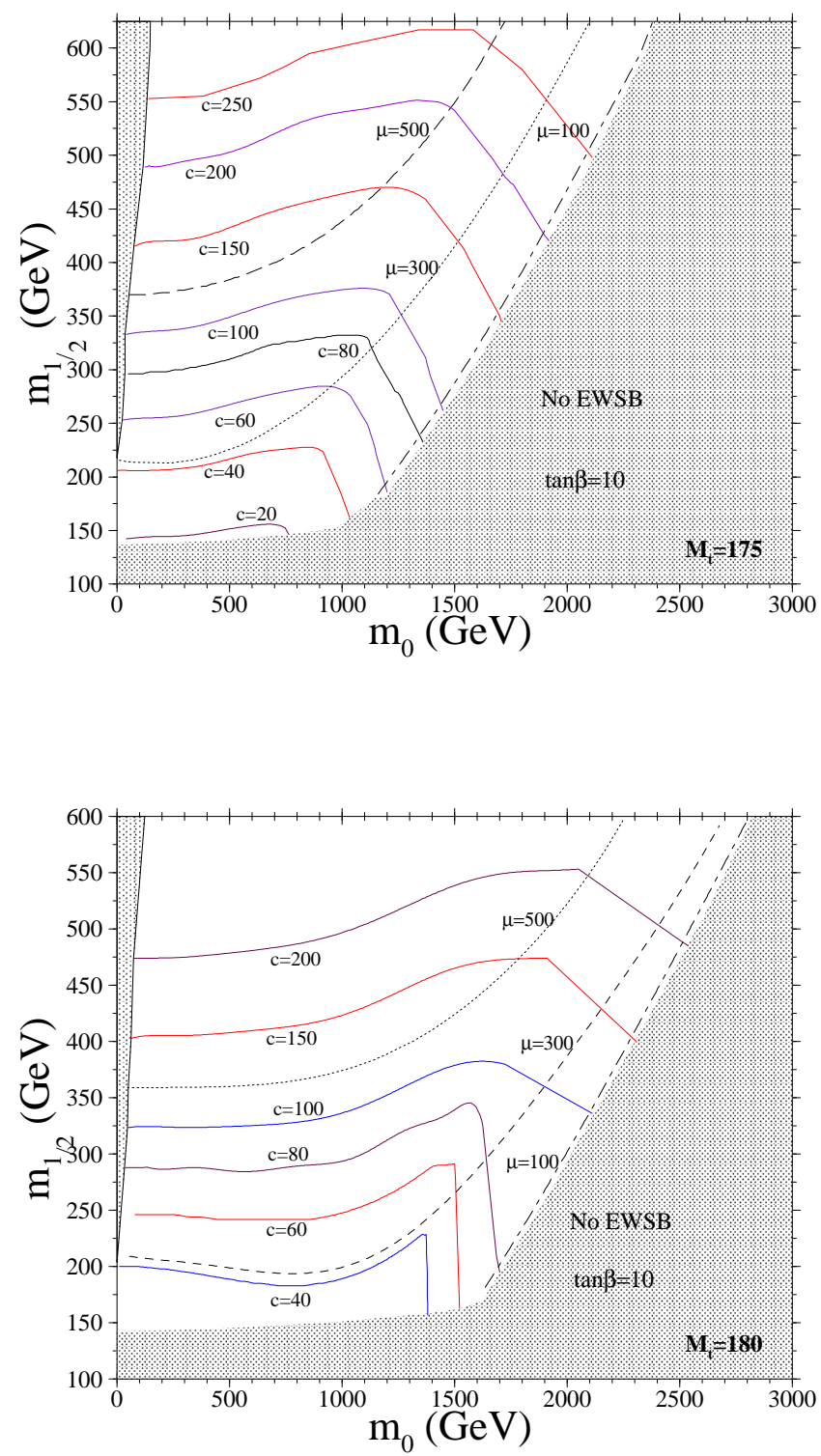

Figure 1: Fine tuning contours for $M_{t}=175$ and $180 \mathrm{GeV}$, with $\mu>0$. Contours of constant $\mu$ are also displayed. Shaded areas in the left sides are the charged LSP regions. 

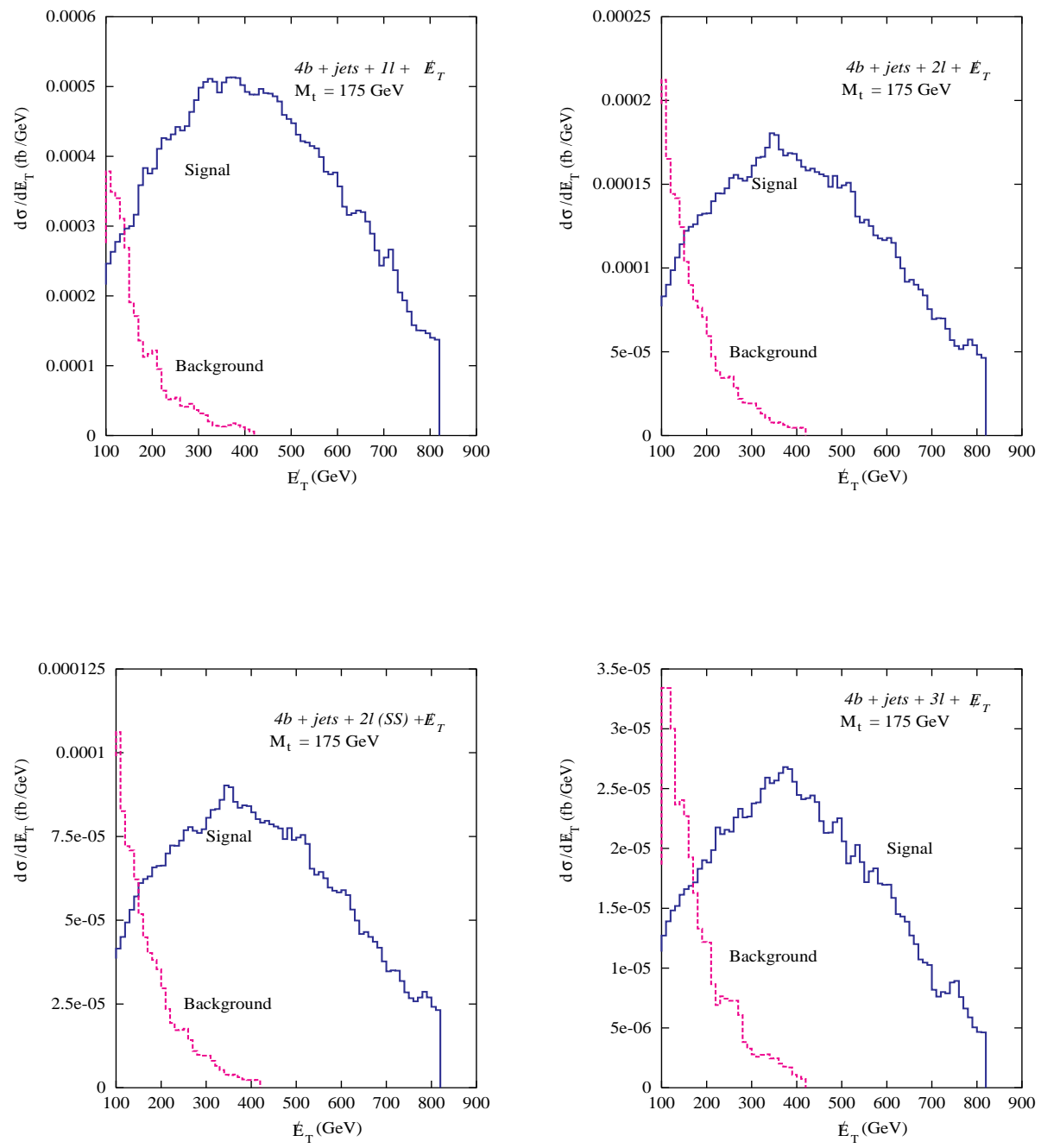

Figure 2: The SUSY signal and the irreducible SM background from $t \bar{t} t \bar{t}$ are shown against the accompanying $\mathscr{E}_{T}$ in the $1-l, 2-l$, same sign $2-l$, and $3-l$ channels with $\geq 3$ b-tags, for $\tan \beta=10$ and $M_{t}=175 \mathrm{GeV}$. 

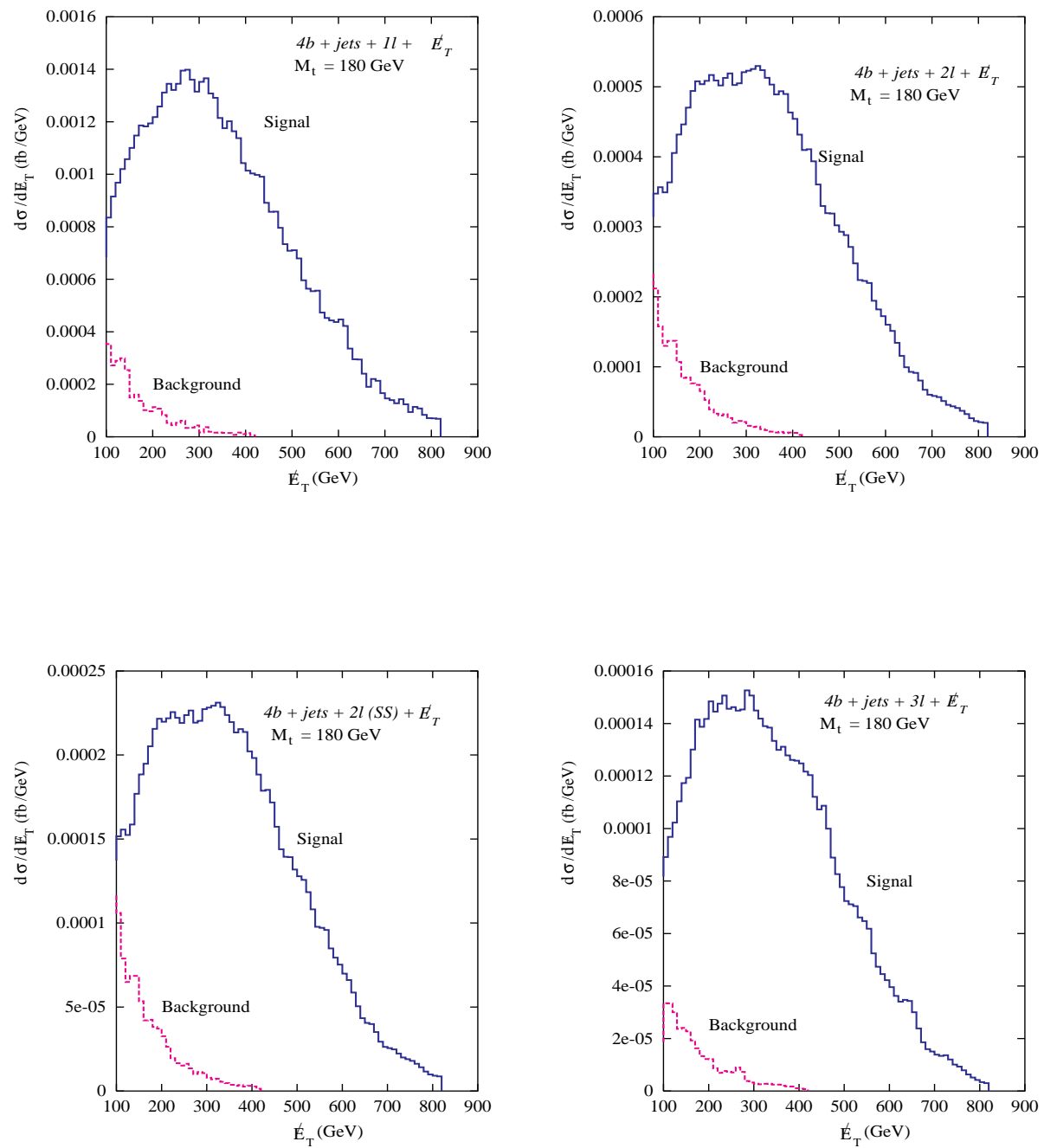

Figure 3: The SUSY signal and the irreducible SM background from $t \bar{t} t \bar{t}$ are shown against the accompanying $\mathscr{E}_{T}$ in the $1-l, 2-l$, same sign $2-l$, and $3-l$ channels with $\geq 3$ b-tags, for $\tan \beta=10$ and $M_{t}=180 \mathrm{GeV}$. 